\title{
Replication-deficient Adenoviral Vector for Gene Transfer Potentiates Airway Neurogenic Inflammation
}

\author{
Giovanni Piedimonte, Raymond J. Pickles, James R. Lehmann, Douglas McCarty, \\ Daniel L. Costa, and Richard C. Boucher \\ Departments of Pediatrics, Medicine, and Pharmacology, University of Miami School of Medicine, Miami, Florida; \\ Cystic Fibrosis/Pulmonary Research and Treatment Center and Gene Therapy Center, The University of \\ North Carolina, Chapel Hill, North Carolina; and United States Environmental Protection Agency, \\ Research Triangle Park, North Carolina
}

\begin{abstract}
Human trials for the treatment of cystic fibrosis lung disease with adenoviral vectors have been complicated by acute inflammatory reactions of unknown etiology. Because replicating respiratory viruses can potentiate tachykinin-mediated neurogenic inflammatory responses in airways, we studied whether the endotracheal administration of a replication-deficient adenoviral vector potentiated this response. The vector Ad5CMVLacZ was administered endotracheally to rats and the leakage of Evans blue dye was used to measure the capsaicin-induced neurogenic albumin extravasation. These studies show that neurogenic albumin extravasation is significantly potentiated in the airways of rats after administration of Ad5CMV$L a c Z$. This inflammatory response can be blocked by selective antagonists of the substance P receptor or by glucocorticoids. Therefore, $(I)$ the acute airway inflammation observed in patients after exposure to adenoviral vectors may exhibit a neurogenic component, which can be blocked pharmacologically, and (2) preclinical adenoviral vector safety studies of other organs innervated by the tachykinin system, e.g., coronary arteries and gastrointestinal tract, should include assessment of neurogenic inflammation. Piedimonte, G., R. J. Pickles, J. R. Lehmann, D. McCarty, D. L. Costa, and R. C. Boucher. 1997. Replication-deficient adenoviral vector for gene transfer potentiates airway neurogenic inflammation. Am. J. Respir. Cell Mol. Biol. 16:250-58.
\end{abstract}

Nonreplicating adenoviral vectors have been used in a large majority of studies designed to transfer functional cystic fibrosis transmembrane conductance regulator (CFTR) to the airway epithelium of cystic fibrosis (CF) patients. Preclinical in vitro studies have revealed efficacy without significant cellular toxicity. However, several studies suggest that the vectors used in the initial human trials in vivo retain significant inflammatory properties of the wild-type virus despite their inability to replicate efficiently.

A recent double-blind vehicle controlled trial of gene transfer in the nasal epithelium of patients with CF reported the acute onset of local mucosal toxicity and increased albumin flux in the nasal lavages of 2 of 3 patients given topical administration of a high titer of an adenoviral vector (1). Sur-

(Received in original form July 17, 1996 and in revised form September 10, 1996)

Address correspondence to: Giovanni Piedimonte, M.D., Pediatric Pulmonary Division, Mailman Center for Child Development, University of Miami School of Medicine, 1601 N.W. 12th Ave., Miami, FL 33136.

Abbreviations: cystic fibrosis, $\mathrm{CF}$; cystic fibrosis transmembrane conductance regulator, CFTR; neurokinin, NK; phosphate-buffered saline, PBS; particles per milliliter, $\mathrm{p} / \mathrm{mL}$; substance $\mathrm{P}, \mathrm{SP}$; ultraviolet, UV.

Am. J. Respir. Cell Mol. Biol. Vol. 16. pp. 250-258, 1997 prisingly, this inflammatory reaction was not associated with leukocyte chemotaxis or with the release of cytokines. Although none of the extensive preclinical studies had focused on the potential for neurogenic-mediated inflammation as being a potential problem with adenoviral vector administration, the timing and characteristics of the vector-induced reactions observed in the human trial were compatible with this type of response, which is caused by the local release of substance P (SP) and other peptide neurotransmitters with proinflammatory properties from unmyelinated sensory nerves (2). These peptidergic nerves are widely distributed in organs throughout the body, including the heart (3), the gastrointestinal tract (4), and the respiratory system (2), and their inflammatory effects can be potentiated by a number of agents, including viruses (5). Therefore, we hypothesized that the sensory nerves in the respiratory mucosa are "primed" by adenoviral vectors and may evoke exaggerated inflammatory responses upon stimulation. If so, the acute inflammatory reactions observed in clinical trials using adenoviral vectors may be mediated, at least in part, by the inflammatory neuropeptides contained in the sensory nerve terminals.

To test this hypothesis, we used a rat model of neurogenic inflammation. Rat tracheas were exposed in vivo to an 
adenoviral vector (Ad5CMVLacZ) similar to that used in clinical trials and stimulated with an intravenous infusion of capsaicin, a drug known to release SP and other peptides from sensory nerves (6). Changes in airway vascular permeability were measured using Evans blue dye as a tracer of albumin extravasation (7). Vector-induced potentiation of neurogenic albumin extravasation was studied after inactivation of the vector nucleic acid by heating or by ultraviolet (UV) light irradiation in the presence of psoralen (8). The specific role of the SP receptors (also known as neurokinin [ $\left.\mathrm{NK}_{1}\right]$ receptors) (9) was explored with exogenous administrations of SP and with the selective receptor antagonist CP-99,994 (10). Finally the anti-inflammatory effect of steroids in this system was assessed by treating rats with dexamethasone or with its vehicle.

\section{Materials and Methods}

\section{Animals}

We used adult male pathogen-free Fischer F344 rats, 12 to $14 \mathrm{wk}$ of age, body weight ranging from 220 to $288 \mathrm{~g}$, from Charles River Breeding Laboratories (Raleigh, NC). To prevent microbial contamination, rats were housed in groups of 2 or 3 in autoclaved plastic cages isolated by filter-tops (Micro-Isolator System, Lab Products Inc., Maywood, NJ). All manipulations were conducted inside a laminar flow workbench (Stay-Clean, Lab Products, Inc.). Experimental procedures followed in this study were approved by the Animal Care and Use Committee of the Health Effect Research Laboratory of the U.S. Environmental Protection Agency.

\section{Preparation of Vector Suspensions}

The adenoviral vector used for these studies, Ad5CMV$L a c Z$, was derived from Addl 309, a mutant strain of adenovirus type 5 which does not express functional E3 19k protein. The LacZ transgene, under the control of the CMV immediate early promoter, was spliced into the left end of the viral genome by homologous recombination, replacing the viral $\mathrm{E} 1$ region. The generation of the adenovirus vector utilized standard protocols for adenovirus overlapping recombination on 293 cells. The titer of the recombinant adenoviral stock was determined by counting X-gal-stained 293 cells. Ad5CMVLacZ used in this study was purified on $\mathrm{CsCl}$ gradients and the buffer containing the vector particles ( $\mathrm{CsCl}$ in $25 \mathrm{mM}$ Tris with $25 \%$ glycerol, $\mathrm{pH} 7.5$ ) was exchanged before each experiment by passing the vector suspensions through G-50 Sephadex columns (Quick Spin, Boehringer Mannheim, Indianapolis, IN) equilibrated in sterile phosphate-buffered saline (PBS). The virus particle number was calculated from the optical density of the suspensions measured at $260 \mathrm{~nm}$ wavelength.

The animals used in this study were dosed with $100 \mu \mathrm{L}$ of PBS suspensions containing $3 \times 10^{12}$ vector particles per $\mathrm{mL}(\mathrm{p} / \mathrm{mL})$, corresponding to $1 \times 10^{11}$ infectious units per $\mathrm{mL}$. Serial dilutions for the dose/response curves were performed by diluting the virus stock suspension in sterile PBS. Dosing was always performed within $2 \mathrm{~h}$ of resuspending the vector in PBS to prevent significant decline in viral titer. Control rats were dosed with sterile PBS.

\section{Vector Inactivation}

Aliquots of Ad5CMVLacZ suspended in a buffer containing $33 \mu \mathrm{g} / \mu \mathrm{L}$ of 8 -methoxypsoralen were irradiated with a 365 $\mathrm{nm}$ UV light source for $30 \mathrm{~min}$ on ice to inactivate the vector nucleic acid (8). Before administration, the UV-treated vector was filtered on G-50 Sephadex columns equilibrated with PBS. The concentrations of viral particles and infectious units were measured before and after UV irradiation by optical density and by X-gal staining of infected 293 cells, respectively. The number of Ad5CMVLacZ particles remained unchanged after UV irradiation, whereas titers in 293 cells decreased by approximately 4 orders of magnitude. In separate experiments, aliquots of Ad5CMVLacZ were heat-inactivated by incubation at $65^{\circ} \mathrm{C}$ for $12 \mathrm{~h}$. No $\mathrm{LacZ}$ gene expression could be detected after exposure of 293 cells to heat-inactivated vector.

\section{Vector Administration}

Rats were anesthetized with sodium pentobarbital $(50 \mathrm{mg} / \mathrm{kg}$ i.p.). The vocal cords were visualized with a laryngoscope, and the trachea carefully intubated with a 16-gauge cannula. An 18-gauge inner cannula, connected to a tuberculin syringe, was passed through the endotracheal cannula to deposit the $100-\mu \mathrm{L}$ inoculum over the airway mucosa between the cricoid cartilage and the first tracheal ring. Preliminary experiments using India ink were done to obtain cannulas of appropriate length and assure the localization of the inoculum to a confined area on the tracheal surface.

\section{Production of Neurogenic Inflammation}

Five days after dosing with vector or vehicle, the rats were anesthetized with sodium pentobarbital. Evans blue dye (30 $\mathrm{mg} / \mathrm{kg} \mathrm{i.v.} \mathrm{over} 5 \mathrm{~s}$ ) was injected to measure the extravasation of albumin associated with the neurogenic inflammation (7). Immediately after the injection of the tracer, sensory nerves were stimulated by an intravenous injection of capsaicin (75 $\mu \mathrm{g} / \mathrm{kg}$ over $2 \mathrm{~min}$ ) (6). Control rats received vehicle used to dissolve the capsaicin ( $1 \mathrm{~mL} / \mathrm{kg} \mathrm{i.v.)}$ to quantitate the baseline extravasation of the tracers in the absence of neural stimulation. Ten rats received an i.v. injection of SP $(1 \mu \mathrm{g} / \mathrm{kg}$ over $20 \mathrm{~s})$.

Five minutes after the injection of the tracer, the chest was opened, a cannula inserted into the ascending aorta through the left ventricle, and the circulation perfused for $2 \mathrm{~min}$ with PBS using a syringe pump set at the rate of $50 \mathrm{~mL} / \mathrm{min}$. The trachea and main-stem bronchi were dissected and cut into an extrathoracic segment (from the first cartilaginous ring to the thoracic inlet) and an intrathoracic segment (from the thoracic inlet to the end of the main-stem bronchi). The specimens were dissected free of connective tissue, opened along the ventral midline, blotted, weighed, and incubated in $1 \mathrm{~mL}$ of formamide (Sigma, St. Louis, MO) at $50^{\circ} \mathrm{C}$ for $18 \mathrm{~h}$ to extract the extravasated Evans blue dye.

\section{Measurement of Albumin Extravasation}

The extravasation of Evans blue-labeled albumin from the tracheal microcirculation was quantified by measuring the optical density of the formamide extracts at a wavelength of $620 \mathrm{~nm}$ (11). The quantity of Evans blue dye extravasated in 
the trachea, expressed in nanograms per milligram of wet weight, was interpolated from a standard curve of Evans blue concentrations $(0.5$ to $10 \mu \mathrm{g} / \mathrm{mL})$.

\section{LacZ Histochemistry}

Five days after the administration of $3 \times 10^{12} \mathrm{p} / \mathrm{mL}$ of Ad5CMVLacZ or sterile PBS ( $n=3$ per group), rats were killed by pentobarbital overdose and their trachea and mainstem bronchi were dissected and rinsed in PBS. The specimens were fixed in $0.5 \%$ glutaraldehyde in PBS, then rinsed twice for $15 \mathrm{~min}$ in PBS containing $1 \mathrm{mM} \mathrm{MgCl}_{2}$ at room temperature. $\beta$-galactosidase activity was detected by staining for $3 \mathrm{~h}$ at $37^{\circ} \mathrm{C}$ with a solution containing X-gal (5-bromo-4chloro-3-indolyl- $\beta$-D-galactopyranoside, $1 \mathrm{mg} / \mathrm{mL}$ ), $5 \mathrm{mM}$ $\mathrm{K}_{3} \mathrm{Fe}(\mathrm{CN})_{6}, 5 \mathrm{mM} \mathrm{K}_{4} \mathrm{Fe}(\mathrm{CN})_{6}$, and $1 \mathrm{mM} \mathrm{MgCl}_{2}$.

\section{Experimental Protocols}

To determine whether the endotracheal administration of an adenoviral vector resulted in an increased neurogenic inflammatory response, 12 rats were dosed with $3 \times 10^{12} \mathrm{p} / \mathrm{mL}$ of Ad5CMVLacZ. A control group of 10 rats was dosed with sterile PBS. Five days after dosing, capsaicin was injected into half of the vector-dosed rats and half of the PBS-dosed rats to stimulate the airway mucosal sensory nerves. The other vector-dosed and PBS-dosed rats received an injection of vehicle. To explore the time course of this inflammatory reaction to adenoviral vectors, 4 groups of rats $(n=5$ per group) dosed with $3 \times 10^{12} \mathrm{p} / \mathrm{mL}$ of Ad5CMVLacZ or with sterile PBS were injected with capsaicin or its vehicle $18 \mathrm{~h}$ after the administration of vector or PBS. To assess the dose dependency of this response, 4 groups of rats $(n=5$ or 6 per group) were dosed with titers ranging from $3 \times$ $10^{4}$ to $3 \times 10^{10} \mathrm{p} / \mathrm{mL}$ of Ad5CMVLacZ and injected with capsaicin 5 days later.

To determine whether this effect of adenoviral vectors was a property of the viral protein capsid of the virus or was dependent upon vector gene expression, 2 groups of rats $(n=$ 6 each) were dosed with $3 \times 10^{12} \mathrm{p} / \mathrm{mL}$ of Ad5CMVLacZ inactivated by heating or by exposure to UV light and psoralen. Both groups were injected with capsaicin 5 days after dosing.

To determine whether the potentiating mechanism of adenoviral vectors on neurogenic inflammation was operating at a pre-synaptic or post-synaptic level, a group of Ad5CMVLacZ-dosed rats and a group of PBS-dosed controls ( $n=5$ each) were injected with SP 5 days after dosing. To determine whether the inflammatory reaction associated with the endotracheal administration of vector can be prevented by selective antagonism of the $\mathrm{SP}\left(\mathrm{NK}_{1}\right)$ receptor, 2 groups of rats $(n=-5$ each) dosed 5 days earlier with $3 \times$ $10^{12} \mathrm{p} / \mathrm{mL}$ of Ad5CMVLacZ were pretreated with the selective $\mathrm{SP}\left(\mathrm{NK}_{1}\right)$ receptor antagonist CP-99,994 (4 mg/kg i.v.), or with its inactive (2R,3R)-enantiomer CP-100,263 (4 mg/ $\mathrm{kg}$ i.v.) $5 \mathrm{~min}$ before the injection of capsaicin $(10,12)$.

Finally, to assess the effect of glucocorticoids on this neurogenic inflammatory response, 2 groups of rats dosed with $3 \times 10^{12} \mathrm{p} / \mathrm{mL}$ of Ad5CMVLacZ were pretreated for 5 days with dexamethasone $(0.5 \mathrm{mg} / \mathrm{kg} /$ day; $n=5)$ or with its vehicle $(1 \mathrm{~mL} / \mathrm{kg} ; n=6)$ given as a single daily i.p. injection
$(7,13)$. The first injection of dexamethasone or vehicle was given within $1 \mathrm{~h}$ after dosing and the last was given on the morning of the experimental day.

\section{Drugs and Chemicals}

All drugs used in this study were delivered in a volume of $1 \mathrm{~mL}$ per $\mathrm{kg}$ of body weight. Evans blue dye was dissolved in $0.9 \% \mathrm{NaCl}$. Capsaicin (8-methyl- $\mathrm{N}$-vanillyl-6-nonenamide; Sigma) was dissolved in a vehicle with a final concentration of $0.75 \%$ ethanol, $0.375 \%$ Tween 80 , and $0.85 \% \mathrm{NaCl}$ in aqueous solution. SP (Sigma) was dissolved in distilled water to obtain the stock solution and then diluted with $0.9 \% \mathrm{NaCl}$ to the final concentration. CP-99,994 [(+)(2S-3S)-3-(2-methoxybenzylamino)-2-phenylpiperidine] and CP-100,263 [(-)-(2R-3R)-3-(2-methoxybenzylamino)-2-phenylpiperidine] were dissolved in $0.9 \% \mathrm{NaCl}$ immediately before

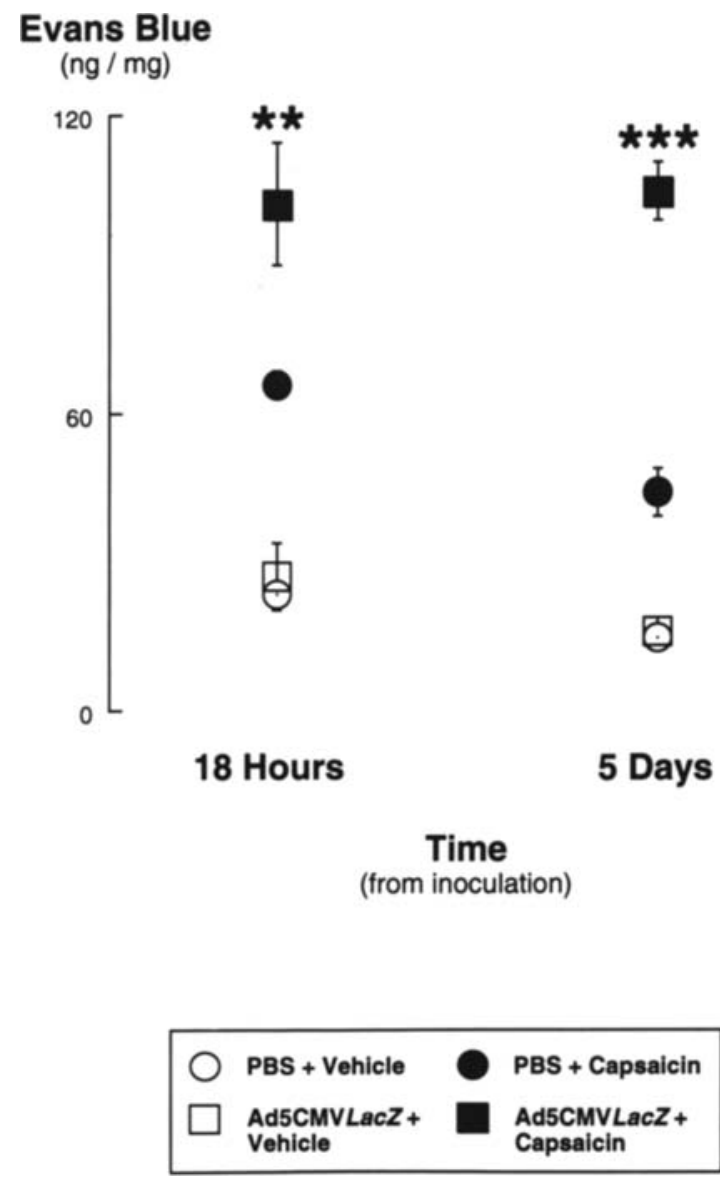

Figure 1. Time dependency of the potentiation of airway neurogenic inflammation caused by endotracheal administration of the adenoviral vector Ad5CMVLacZ $\left(3 \times 10^{12} \mathrm{p} / \mathrm{mL}\right)$. Capsaicininduced extravasation of Evans blue-labeled albumin was significantly larger than in PBS-dosed controls $18 \mathrm{~h}$ after the administration of vector. The difference between vector-dosed and PBS-dosed rats increased 5 days after administration. ${ }^{* *} P<0.01$, $* * * P<0.001=$ significantly different from PBS-dosed control rats. 


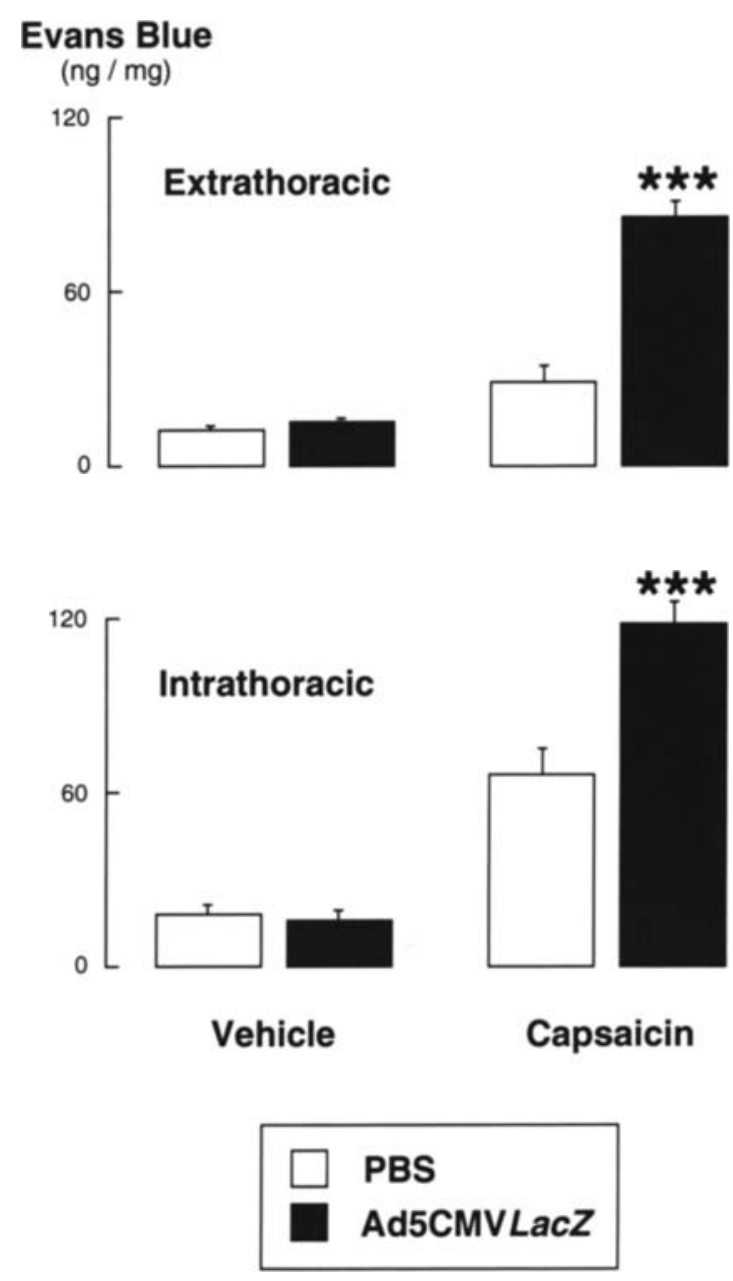

Figure 2. Comparison of the effect of capsaicin on albumin extravasation in the extrathoracic and intrathoracic airway of rats 5 days after a localized endotracheal administration of Ad5CMVLacZ $\left(3 \times 10^{12} \mathrm{p} / \mathrm{mL}\right)$ or PBS. The inoculum was carefully deposited over the airway mucosa between the cricoid cartilage and the first tracheal ring. Significant potentiation of neurogenic albumin extravasation was observed both in the extrathoracic and intrathoracic airway segments. $* * * P<0.001=$ significantly different from PBSdosed control rats.

administration. Dexamethasone sodium phosphate (American Regent Inc., Shirley, NY) was dissolved in a vehicle containing sodium sulfite anhydrous, $1 \mathrm{mg} / \mathrm{mL}$; sodium citrate anhydrous, $19.4 \mathrm{mg} / \mathrm{mL}$; and benzyl alcohol, $1 \% \mathrm{vol} / \mathrm{vol}$; $\mathrm{pH} 7.4$ ).

\section{Statistical Analysis}

Data are expressed as the mean \pm standard error of the mean (SEM). The effects of Ad5CMVLacZ and sensory nerve stimulation on mean values of Evans blue extravasation were analyzed by two-factor analysis of variance (14). Multiple comparisons between means were performed with the Fisher Protected Least Significant Difference test (15). Differences of $P<0.05$ were considered significant.

\section{Results}

In rats dosed with $3 \times 10^{12} \mathrm{p} / \mathrm{mL}$ of Ad5CMVLacZ, cap- saicin-induced Evans blue extravasation $18 \mathrm{~h}$ after dosing was significantly larger than in PBS-dosed controls (Figure 1). Injection of the vehicle of capsaicin induced little extravasation of Evans blue-labeled albumin in the airways of rats dosed with either Ad5CMVLacZ or with sterile PBS. A larger difference in albumin leakage between vector-dosed rats and controls was measured 5 days after dosing. Because this larger difference reflected a decrease in the vehicle-associated extravasation, we chose this time point for routine studies. Vector-mediated potentiation of neurogenic albumin extravasation 5 days after dosing was observed both in the extrathoracic airway, where the inoculum was originally delivered, and in the intrathoracic airway including the mainstem bronchi (Figure 2).

The adenoviral vector potentiated in a dose-dependent fashion the increase in albumin extravasation produced by sensory nerve stimulation with capsaicin (Figure 3). This effect was significant after administration of a vector titer as low as $3 \times 10^{6} \mathrm{p} / \mathrm{mL}$. Heat inactivation and UV-psoralen inactivation of the vector nucleic acid significantly reduced the potentiating effect of Ad5CMVLacZ on capsaicininduced neurogenic inflammation in the rat airways (Figure 4). However, the effect of capsaicin after administration of inactivated vectors was still significantly larger than in control rats dosed with PBS (heat inactivation, $P=0.003$; UVpsoralen inactivation, $P=0.040$ ).

The leakage of Evans blue-labeled albumin induced by intravenous administration of SP, the principal peptide released from sensory nerves, was significantly increased in rats dosed with Ad5CMVLacZ compared with controls

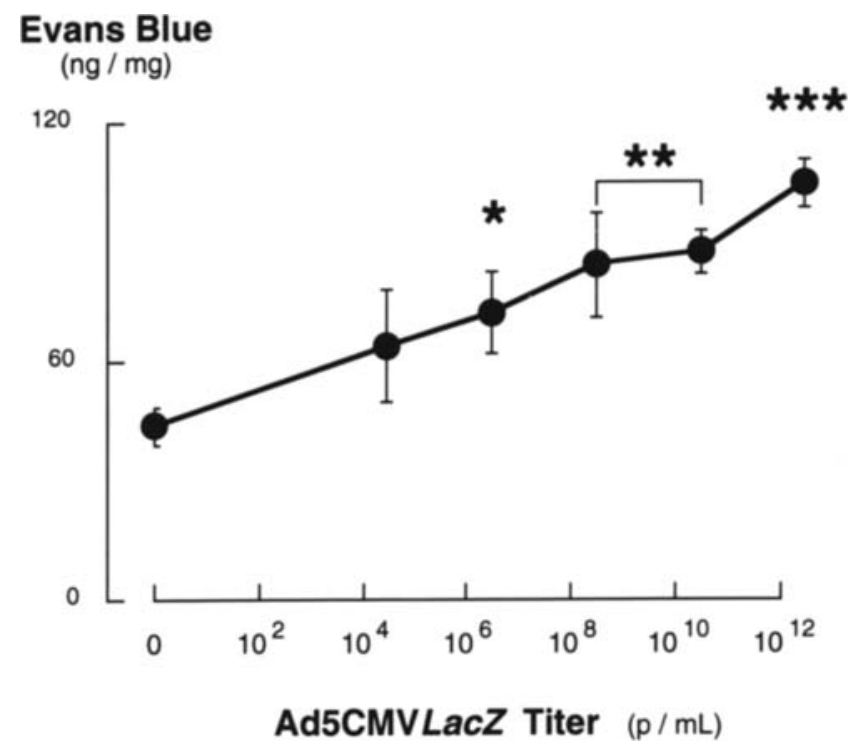

Figure 3. Dose dependency of the potentiation of airway neurogenic inflammation caused by endotracheal administration of Ad5CMVLacZ. Capsaicin-induced extravasation of Evans bluelabeled albumin was significantly larger than in PBS-dosed controls with vector titers as low as $3 \times 10^{6} \mathrm{p} / \mathrm{mL}$. Thus, the threshold of this adverse effect of adenoviral vectors precedes by several orders of magnitude the expression of transgenes in the airway epithelium of rodents. $* P<0.05, * * P<0.01,{ }^{* * * P}<0.001=$ significantly different from PBS-dosed control rats. 

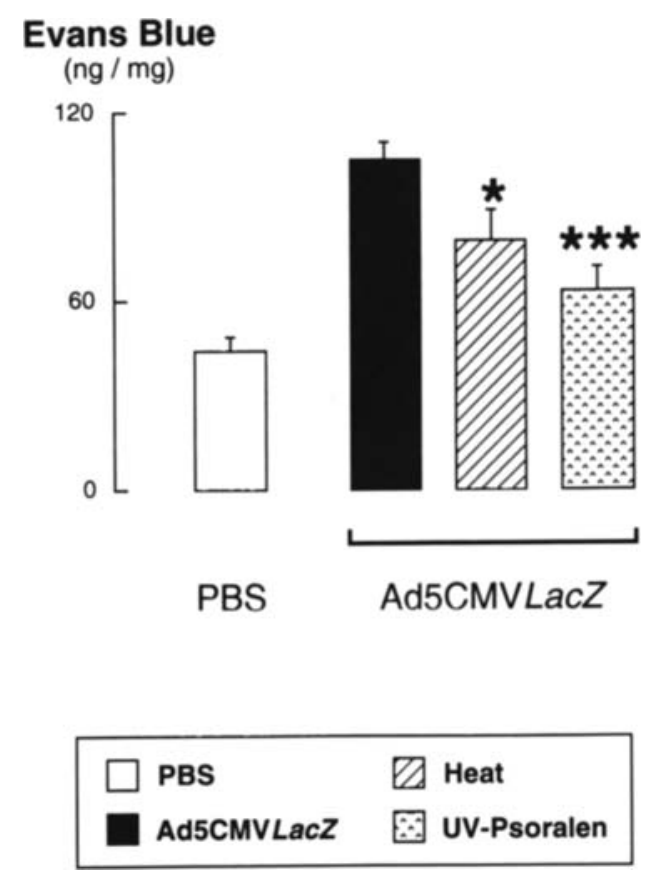

Figure 4. Effect of nucleic acid inactivation on the potentiation of neurogenic inflammation induced by the adenoviral vector Ad5CMV LacZ. Both heat- and UV-psoralen inactivation significantly reduced the potentiating effect of the vector on capsaicin-induced albumin extravasation. However, the effect of capsaicin after administration of inactivated vector was still larger than in control rats dosed with PBS, suggesting that the effect of adenoviral vectors on neurogenic inflammation is caused both by the viral capsid and by expression of vector genes. $* P<0.05, * * * P<0.001=$ significantly different from rats dosed with active Ad5CMVLacZ.

$(84.1 \pm 6.9$ versus $53.8 \pm 7.9 \mathrm{ng} / \mathrm{mg} ; n=5$ rats per group; $P<0.05$ ). However, the magnitude of this potentiation was smaller than that observed with the release of endogenous neuropeptides by capsaicin (see Figures 1-4). The extravasation of Evans blue-labeled albumin produced by capsaicin in rats dosed with Ad5CMVLacZ was fully blocked by the $\mathrm{SP}\left(\mathrm{NK}_{1}\right)$ receptor antagonist CP-99,994, but not by its $(2 \mathrm{R}$, 3R)-enantiomer CP-100,263 (Figure 5). In addition, capsaicin-induced Evans blue extravasation in rats dosed with Ad5CMVLacZ was inhibited by treatment with dexamethasone, but not by its vehicle (Figure 6). After treatment with dexamethasone, capsaicin-induced extravasation in vectordosed rats was not different from that in PBS-dosed rats $(P>0.05)$.

Histochemistry for $\beta$-galactosidase activity in the airways of rats dosed with $3 \times 10^{12} \mathrm{p} / \mathrm{mL}$ of Ad5CMVLacZ 5 days earlier (Figure 7) showed high levels of gene transfer only in the larynx, corresponding to the area of mucosa damaged by the tip of the endotracheal cannula used for the administration of vector. In the rest of the airway, LacZ expression was sparse: small, patchy areas of transduction were observed in the trachea as well as in the main-stem bronchi. No evidence of $\beta$-galactosidase activity was found in the airways of control rats dosed with PBS (not shown).

\section{Discussion}

In a recently completed double-blind vehicle-controlled
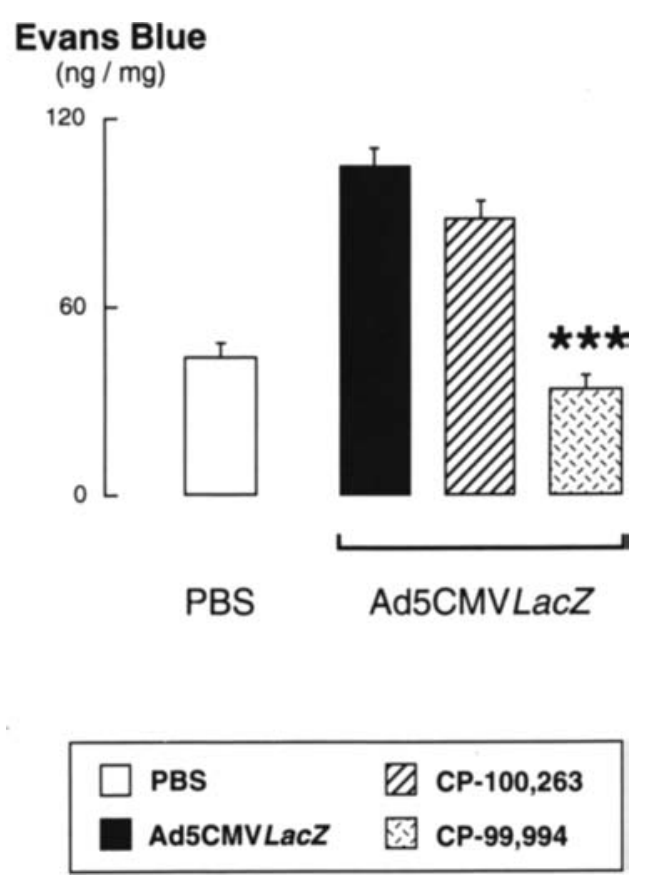

Figure 5. Effect of selective antagonism of the SP(NK $)_{1}$ tachykinin receptor on the potentiation of neurogenic inflammation induced by the adenoviral vector Ad5CMVLacZ. The extravasation of Evans blue-labeled albumin produced by capsaicin in rats dosed with Ad5CMVLacZ was inhibited by CP-99,994, but not by its (2R,3R)-enantiomer CP-100,263. Thus, the potentiation of neurogenic inflammation caused by the administration of Ad5CMVLacZ involves activation of the $\mathrm{SP}\left(\mathrm{NK}_{1}\right)$ receptor and can be prevented by the selective antagonism of this receptor. $* * * P<0.001=$ significantly different from rats with no pretreatment.

study of adenoviral CFTR vectors in the nasal cavity of CF subjects (1), 2 of 3 patients receiving the highest dose of vector experienced a local mucosal inflammatory response confined to the vector-dosed nostril. This adverse response occurred early (within 12-24 h after dosing) and was characterized by mucosal swelling, increased secretions, and albumin leakage. Serial nasal lavages failed to demonstrate an increase in the concentration of inflammatory cytokines or leukocyte chemotaxis; however, a greater increase in lavage cytokines and leukocytes was observed after a mucosal biopsy on the side dosed with the vector, suggesting a "priming" effect.

The timing and the characteristics of vector-mediated inflammatory reactions appeared compatible with a neurogenic-mediated inflammatory response (2). Neurogenic inflammation is evoked by the stimulation of C-type unmyelinated fibers in the respiratory mucosa, which results in the local release of peptide neurotransmitters. The tachykinin SP is the best known of these neuropeptides, and has been shown in a large variety of studies to have potent pro-inflammatory effects in the airways. In particular, exogenously delivered SP is known to increase airway vascular petmeability, thereby causing plasma extravasation and mucosat edema (16). The sensory nerves of the upper (17) and lower (18) respiratory tract of several species, including humans, contain SP-immunoreactive fibers in the airway epithelium and smooth muscle, and around blood vessels. SP-containing 

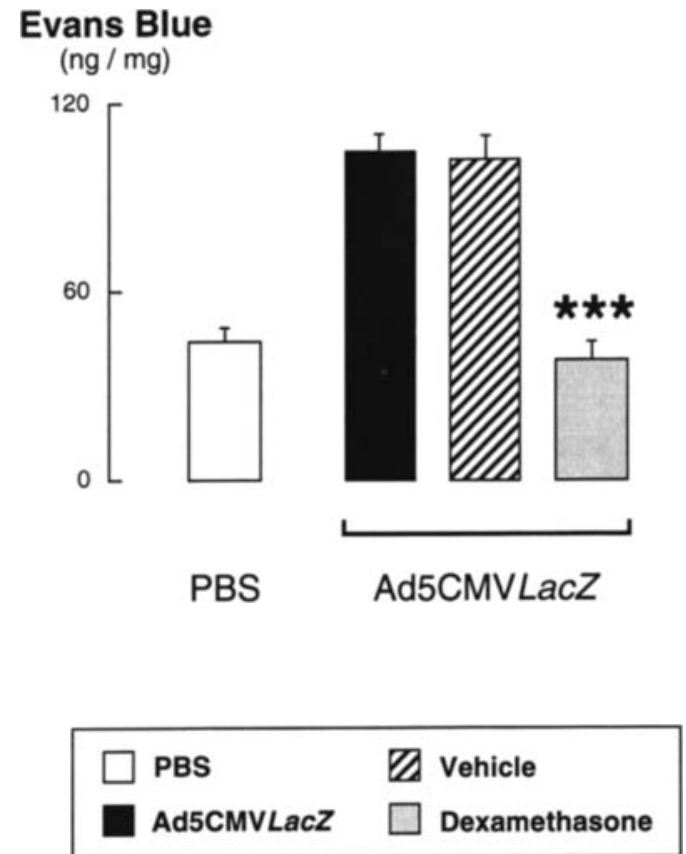

Figure 6. Effect of glucocorticoids on the potentiation of neurogenic inflammation induced by the adenoviral vector Ad5CMVLacZ. The extravasation of Evans blue-labeled albumin produced by capsaicin in rats dosed with Ad5CMVLacZ was inhibited by treatment with dexamethasone, but not by its vehicle. ${ }^{* * *} P<0.001=$ significantly different from rats with no pretreatment

nerves have been identified in several other organs, including the heart (3) and the gastrointestinal tract (4). Release of SP from these nerves can be induced in experimental models by chemical stimulation of the nerves with capsaicin (6).

\section{Adenoviral Vector-mediated Neurogenic Inflammation}

Our data indicate that the localized endotracheal administration of a nonreplicating adenoviral gene transfer vector potentiates neurogenic inflammation in the respiratory tract of rats, as reflected by an abnormally large increase in vascular permeability in response to sensory nerve stimulation by capsaicin. Airway vascular permeability is not affected by the vector in the absence of chemical nerve stimulation. This effect of adenoviral vectors exhibits an early onset, being detectable within $18 \mathrm{~h}$ from vector administration (Figure 1). A larger difference in albumin leakage between vector-dosed rats and controls was measured 5 days after dosing, which may reflect some mucosal damage produced by the endotracheal intubation that affects vascular permeability in the control rats at $18 \mathrm{~h}$ but resolves by 5 days. Although the inoculum was deposited over a confined area of the upper tracheal mucosa, potentiation of neurogenic albumin extravasation was measured in the intrathoracic airway, including the lower trachea and main-stem bronchi, as well as in the extrathoracic airway (Figure 2). This result could be produced by a fraction of the inoculum reaching the lower airway, or by the activation of secondary mechanisms propagating the inflammatory process across the airway mucosa.

The Ad5CMVLacZ vector potentiates in a dose-depen- dent fashion the increase in plasma extravasation produced by sensory nerve stimulation with capsaicin (Figure 3 ). This effect is significant with a vector titer as low as $3 \times 10^{6} \mathrm{p} /$ $\mathrm{mL}$. Because of the inefficiency of adenoviral-mediated gene transfer in vivo (19), the threshold dose for this adverse effect of adenoviral vectors is several orders of magnitude below that necessary for the expression of transgenes in the normal airway epithelium of rodents and humans. Because the effects of neurogenic inflammation may vary among species, it is difficult to extrapolate our data to the human airway without specific clinical studies. However, the evidence presented in this study warrants the inclusion of measurements of neurogenic inflammation in human protocols exploring the safety of future gene therapy vectors.

Previous studies in rats with wild-type murine parainfluenza type I (Sendai) virus revealed that viral respiratory infections strongly potentiate the increase in permeability of tracheal blood vessels and the adherence of neutrophils to the vascular endothelium produced by capsaicin-induced neurogenic inflammation $(5,7)$. This effect was observed 6 days after the administration of virus, when the pathologic changes due to viral replication are maximal. One of the mechanisms responsible for this potentiation seemed to be the loss of epithelial cells rich in neutral endopeptidase, a peptide-degrading enzyme that modulates the biological activity of SP and other tachykinins (20). This enzymatic activity is concentrated in the basal layer of the respiratory epithelium and is reduced in the airways of rodents with viral respiratory infections (21-23). The data shown in the present study suggest that the potentiation of neurogenic inflammation associated with the presence of viral particles in the respiratory tract is independent of their ability to replicate and cause cellular damage. Thus, it is likely that additional mechanisms, independent of the activity of peptide-degrading enzymes, are involved in the mechanism of virus-induced potentiation of neurogenic inflammation.

\section{Mechanism of Potentiation}

We sought to determine whether the potentiating effect of Ad5CMVLacZ on neurogenic inflammation was dependent upon the expression of vector genes, or was rather a property of the adenoviral protein capsid. Inactivation of the vector nucleic acid by heating or by irradiation with UV light in the presence of psoralen significantly reduced the effect of vector particles on neurogenic inflammation (Figure 4). These results suggest that the proinflammatory effect of the adenoviral vector is linked in part to the expression of vector DNA in the respiratory epithelium. However, a significant component of this effect can still be detected after inactivation of the vector nucleic acid, and thus seems to be associated with a direct toxic effect of the adenoviral capsid. Further data describing the relative activity of different components of adenoviral vectors require studies of the neurogenic inflammatory effects of purified capsid proteins (fiber, penton, hexon) and adenoviral mutants with selective deletions of the viral genome.

Adenoviral vectors can produce their effect at different levels in the complex neurogenic inflammatory pathway (2). The mechanism of potentiation could be pre-synaptic (e.g., increased neuropeptide content of nerve terminals) or could 
Figure 7. Histochemical preparations representative of the efficiency of transduction of the $L a c Z$ reporter gene in the airway epithelium of rats dosed with $3 \times$ $10^{12} \mathrm{p} / \mathrm{mL}$ of Ad5CMVLacZ 5 days earlier. High levels of transduction were observed only in the larynx, corresponding to the area of respiratory mucosa damaged by the tip of the endotracheal cannula used for the administration of vector (panel $A$ ). In the rest of the airway, the pattern of $L a c Z$ expression was sparse: small, patchy areas of transduction were observed in the trachea $($ panel $B)$ as well as in the main-stem bronchi. Scale bars: $250 \mu \mathrm{m}$.
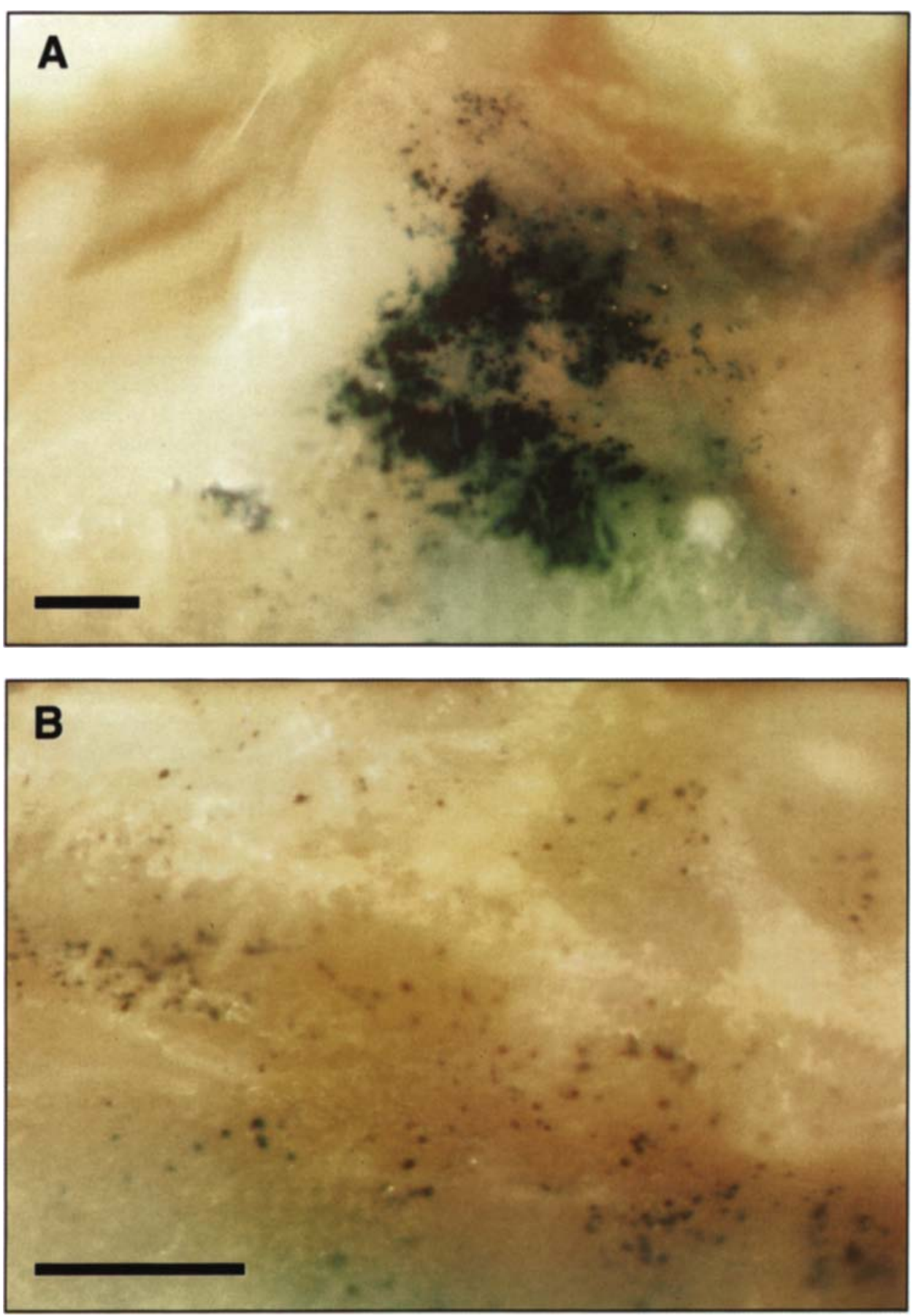

involve one or more post-synaptic levels: decreased peptidedegrading activity, increased density and/or affinity of specific receptors, modification in the intracellular signal transduction following the ligand-receptor interaction. In our study, the albumin leakage caused by SP $(1 \mu \mathrm{g} / \mathrm{kg}$ i.v. $)$, the principal peptide released from capsaicin-sensitive sensory nerves, was significantly increased in rats dosed with Ad5CMVLacZ compared with controls. However, the magnitude of this potentiation was smaller than that observed with the re- lease of endogenous neuropeptides by capsaicin. This observation indicates that the vector exerts at least part of its effect at the post-synaptic level. The difference between SP and capsaicin may be due to post-synaptic potentiation of other tachykinins, e.g., neurokinins A (NKA) and B (NKA) coreleased with SP, or to pre-synaptic modifications affecting the sensory nerves. It is also possible that the concentration of injected SP reaching the receptors is lower than that released endogenously upon stimulation of sensory nerves. 


\section{Pharmacologic Modulation of}

\section{Vector-induced Inflammation}

Three mammalian NK receptors have been cloned and their protein sequence with 7 putative transmembrane spanning helices typical of G protein-coupled receptors defined (24). Each of the tachykinin peptides can act as a full agonist on each of the 3 receptors if present at sufficiently high concentrations. However, SP, NKA, and NKB display preferential affinities for $\mathrm{NK}_{1}, \mathrm{NK}_{2}$, and $\mathrm{NK}_{3}$ receptors, respectively (9). In our study, selective antagonism of the $\mathrm{SP}\left(\mathrm{NK}_{1}\right)$ receptor with CP-99,994 $(10,12)$ abolished the effect of Ad5CMVLacZ on airway neurogenic inflammation (Figure 5). The (2R,3R)-enantiomer CP-100,263, which shares the same pharmacologic properties of CP-99,994 but is inactive on the $\operatorname{SP}\left(\mathrm{NK}_{t}\right)$ receptor, did not produce inhibition, confirming the specificity of the effect of CP-99,994.

Dexamethasone has been shown to prevent the potentiation of neurogenic inflammation caused by replicating viruses in the respiratory tract (7). The neurogenic inflammatory effect of Ad5CMVLacZ was also abolished by treatment with a relatively low dose of this glucocorticoid (Figure 6). Glucocorticoids can inhibit the neurogenic inflammatory cascade at multiple levels: upregulation of peptidase activity $(13,25)$, inhibition of $\mathrm{NK}_{1}$ receptor mRNA transcription (26), and decrease in the SP content of dorsal root ganglia (27). The inhibitory effect of dexamethasone on SP-induced extravasation in the rat airways was observed with low doses that did not affect the extravasation induced by the nonpeptide inflammatory mediator platelet activating factor (13). Thus the airway neurogenic inflammation caused by adenoviral vectors in the respiratory tract possibly can be reduced with low doses of systemic or inhaled glucocorticoids.

This study shows that a replication-deficient adenoviral vector similar to those used in clinical trials of gene therapy for CF renders the airways of rats abnormally susceptible to neurogenic inflammation. This adverse effect of adenoviral vectors has an early onset and requires relatively low titers. The potentiation seems to involve both pre- and postsynaptic levels of the neurogenic inflammatory pathway and to be caused in part by vector gene expression and in part by a direct toxic effect of the viral capsid. The neurogenicmediated extravasation of albumin involves activation of the $\mathrm{SP}\left(\mathrm{NK}_{1}\right)$ receptor and can thus be prevented by the selective antagonism of this receptor or by the use of low-dose glucocorticoids. The potentiation of neurogenic-mediated airway inflammation in response to replication-deficient viral vectors may contribute to the pulmonary inflammatory reaction to adenovirus-derived CFTR vectors observed in humans. If so, pharmacologic modulation of this inflammatory pathway, e.g., receptor antagonists or glucocorticoids, may minimize the acute toxic effects of adenoviral vectors and perhaps allow the safe administration of the higher titers necessary to achieve therapeutic effects. Further testing will be required to evaluate whether these principles will apply to other organs innervated by $\mathrm{C}$-fibers that are targets for adenovirus-mediated gene transfer.

Acknowledgments: The authors thank Dr. John A. Lowe, III (Pfizer Central Research Division) for kindly providing the NK receptor antagonist CP-99,994 and its enantiomer CP-100,263. Some of the findings reported in this paper were presented at the 9th Annual North American CF Conference and at the 1996
ALA/ATS International Conference. The research described in this article has been reviewed by the National Health and Environmental Effects Research Laboratory, U.S. Environmental Protection Agency, and approved for publication Approval does not signify that the contents necessarily reflect the views and the policies of the Agency nor does mention of trade names or commercial products constitute endorsement or recommendation for use.

\section{References}

I. Knowles, M. R., K. W. Hohneker, Z. Zhou, J. C. Olsen, T. L. Noah, P.-C Hu, M. W. Leigh, J. Engelhardt, L. J. Edwards, K. R. Jones, M. Gross man, J. M. Wilson, L. G. Johnson, and R. C. Boucher. 1995. A controlled study of adenoviral-vector-mediated gene transfer in the nasal epithelium of patients with cystic fibrosis. $N$. Engl. J. Med. 333:823-831.

2. Piedimonte, G. 1995. Tachykinin peptides, receptors, and peptidases in airway disease. Exp. Lung Res. 21:809-834

3. Weihe, E., M. Reinecke, D. Opherk, and W. G. Forssmann. 1981. Peptidergic innervation (substance P) in the human heart. J. Mol. Cell Cardiol. 13:331-333

4. Costa, M., J. B. Furness, I. J. Llewellyn-Smith, R. Murphy, J. C. Bornstein and J. R. Keast. 1985. Functional roles for substance P-containing neurons in the gastrointestinal tract. In Substance P Metabolism and Biological Actions. C. C. Jordan and P. Oehme, editors. Taylor \& Francis, London. 99-119.

5. Piedimonte, G., J. A. Nadel, E. Umeno, and D. M. McDonald. 1990. Sendai virus infection potentiates neurogenic inflammation in the rat trachea. J. Appl. Physiol. 68:754-760.

6. Holzer, P. 1991. Capsaicin: cellular targets, mechanisms of action, and selectivity for thin sensory neurons. Pharmacol. Rev. 43:143-201

7. Piedimonte, G., D. M. McDonald, and J. A. Nadel. 1990. Glucocorticoids inhibit neurogenic plasma extravasation and prevent virus-potentiated extravasation in the rat trachea. J. Clin. Invest. 86:1409-1415.

8. Cotten, M., E. Wagner, K. Zatloukal, S. Phillips, D. T. Curiel, and M. L. Birnstiel. 1992. High-efficiency receptor-mediated delivery of small and large 48 kilobase gene constructs using the endosome-disruption activity of defective or chemically inactivated adenovirus particles. Proc. Natl. Acad. Sci. USA 89:6094-6098.

9. Regoli, D., G. Drapeau, S. Dion, and R. Couture. 1988. New selective agonists for neurokinin receptors: pharmacological tools for receptor characterization. Trends Pharmacol. Sci. 9:290-295.

10. McLean, S., R. M. Snider, M. C. Desai, T. Rosen, D. K. Bryce, K. P. Longo, A. W. Schmidt, and J. Heym. 1993. CP-99,994, a nonpeptide antagonist of the tachykinin NK, receptor. Regul. Pept. 46:329-331.

11. Saria, A, J. M. Lundberg, G. Skofitsch, and F. Lembeck. 1983. Vascular protein leakage in various tissues induced by substance P, capsaicin, bradykinin, serotonin, histamine, and by antigen challenge. Naunyn Schmiedebergs Arch. Pharmacol. 324:212-218.

12. Piedimonte, G., C. Bertrand, P. Geppetti, R. M. Snider, M. C. Desai, and J. A. Nadel. 1993. A new NK, receptor antagonist (CP-99,994) prevents the increase in tracheal vascular permeability produced by hypertonic saline. J. Pharmacol. Exp. Ther. 266:270-273.

13. Piedimonte, G., D. M. McDonald, and J. A. Nadel. 1991. Neutral endopeptidase and kininase II mediate glucocorticoid inhibition of neurogenic inflammation in the rat trachea. J. Clin. Invest. 88:40-44.

14. $\mathrm{Zar}, \mathrm{J}, \mathrm{H} .1984$. Two-factor analysis of variance. In Biostatistical Analysis Prentice-Hall, Inc., Englewood Cliffs, New Jersey. 206-235.

15. Wallenstein, S., C. L. Zucker, and J. L. Fleiss. 1980. Some statistical methods useful in circulation research. Circ. Res. 47:1-9.

16. Lundberg, J. M., and A. Saria. 1982. Capsaicin-sensitive vagal neurons involved in control of vascular permeability in tat trachea. Acta Physiol. Scand. 115:521-523

17. Baraniuk, J. N., J. D. Lundgren, J. Mullol, M. Okayama, M. Merida, and M. Kaliner. 1991. Substance $P$ and neurokinin $A$ in human nasal mucosa Am. J. Respir. Cell Mol. Biol. 4:228-236.

18. Lundberg, J. M., T. Hokfelt, C.-R. Martling, A. Saria, and C. Cuello 1984. Substance P-immunoreactive sensory nerves in the lower respiratory tract of various mammals including man. Cell Tissue Res. 235:251-261.

19. Grubb, B. R., R. J. Pickles, H. Ye, J. R. Yankaskas, R. N. Vick, J. F Engelhardt, J. M. Wilson, L. G. Johnson, and R. C. Boucher. 1994. Inefficient gene transfer by adenovirus vector to cystic fibrosis airway epithelia of mice and humans. Nature 371:802-806

20. Nadel, J A. 1992. Regulation of neurogenic inflammation by neutral endopeptidase. Am. Rev. Respir. Dis. 145:S48-S52.

21. Jacoby, D. B., J. Tamaoki, D. B. Borson, and J. A. Nadel. 1988. Influenza infection causes airway hyperresponsiveness by decreasing enkephalinase. J. Appl. Physiol. 64:2653-2658.

22. Dusser, D., D. Jacoby, T. Djokic, I. Rubinstein, D. Borson, and J. Nadel. 1989. Virus induces airway hyperresponsiveness to tachykinins: role of neutral endopeptidase. J. Appl. Physiol. 67:1504-151 I. 
23. Borson, D., J. Brokaw, K. Sekizawa, D. McDonald, and J. Nadel. 1989. Neutral endopeptidase and neurogenic inflammation in rats with respiratory infections. J. Appl. Physiol. 66:2653-2658.

24. Nakanishi, S. 1991. Mammalian tachykinin receptors. Ann. Rev. Neurosci. 14:123-136.

25. Borson, D. B., and D. C. Gruenert. 1991. Glucocorticoids induce neutral endopeptidase in transformed human tracheal epitheljal cells. Am. J. Physiol. 260(Lung Cell. Mol. Physiol. 4):L83-L89.
26. Thara, H., and S. Nakanishi. 1990. Selective inhibition of expression of the substance $P$ receptor $m R N A$ in pancreatic acinar $A R 42 J$ cells by glucocorticoids. J. Biol. Chem. 265:22441-22445.

27. Smith, G. D., J. R. Seckl, W. J. Sheward, J. G. Bennie, S. M. Carrol, H. Dick, and A. J. Harmar. 1991. Effect of adrenalectomy and dexamethasone on neuropeptide content of dorsal root ganglia in the rat. Brain Res. $564: 27-30$ 